BULL. AUSTRAL. MATH. SOC.

VOL. 31 (1985), 329-338.

\title{
ON THE DISCREPANCY OF THE SEQUENCE FORMED FROM MULTIPLES OF AN IRRATIONAL NUMBER
}

\author{
Tony van Ravenstein
}

\begin{abstract}
This paper demonstrates a connection between two measures of discrepancy of sequences which arise in the theory of uniform distribution modulo one. The sequence formed from the nonnegative integer multiples of an irrational number $\xi$ is investigated and, by an application of the "Steinhaus Conjecture", some values of the two discrepancies are obtained using continued fractions.
\end{abstract}

\section{Introduction}

Let $v=\left(x_{1}, x_{2}, x_{3}, \ldots\right)$ be an infinite sequence of real numbers located in the unit interval. We define the standard discrepancy of the first $N$ terms of $v$ as

$$
D_{N}(\nu)=\sup _{0 \leq \alpha \leq \beta<1}\left|\frac{A([\alpha, \beta] ; \nu ; N)}{N}-(\beta-\alpha)\right|,
$$

where $A([\alpha, \beta] ; \nu ; N)$ counts the number of the first $N$ elements of $\nu$ which belong to $[\alpha, \beta]$.

The discrepancy is a measure of how closely the first $N$ elements of $\nu$ approximate a uniform distribution. A related measure (the extreme

Received 30 October 1984. The author is most grateful to Dr Keith Tognetti of the University of Wollongong for helpful discussions.

Copyright Clearance Centre, Inc. Serial-fee code: 0004-9727/85 $\$ A 2.00+0.00$. 
discrepancy where $\alpha=0$ ) is given by

$$
D_{N}^{*}(\nu)=\sup _{0 \leq \beta<1}\left|\frac{A([0, \beta] ; \nu ; N)}{N}-\beta\right| .
$$

In this paper we first demonstrate an interesting relationship between these two measures. In particular, we consider the sequence $\omega$ formed by the fractional parts of non-negative integer multiples of $\xi$, where $\xi$ is an irrational number. $D_{N}(\omega)$ and $D_{N}^{*}(\omega)$ are then evaluated for some particular values of $N$ based on the continued fraction expansion of $\xi$. The formula largely derives from a result related to the "Steinhaus Conjecture" (see Section 5). An upper bound of $\underset{N \rightarrow \infty}{\lim \inf N D_{N}(\omega)}$ is offered as a corollary.

\section{Representation of $v$ on a circle}

Suppose that the sequence $v$ is represented on the circle $C$ of unit circumference, rather than on the unit interval. Let $[\alpha: \beta]$ denote the arc from $\alpha$ to $\beta \quad(0 \leq \alpha, \beta<1)$ on the circle in the direction of increasing co-ordinate. That is,

$$
[\alpha: \beta]= \begin{cases}{[\alpha, \beta],} & 0 \leq \alpha \leq \beta<1, \\ C-(\beta, \alpha), & 0 \leq \beta<\alpha<1 .\end{cases}
$$

Note that the length of such an arc is equal to $\{\beta-\alpha\}$, the fractional part of $\beta-\alpha$.

With respect to this representation, the discrepancy of $\nu$ may be given by

$$
\hat{D}_{N}(\nu)=\sup _{0 \leq \alpha, \beta<1}\left|\frac{A([\alpha ; \beta] ; \nu ; N)}{N}-\{\beta-\alpha\}\right| .
$$

LEMMA. $D_{N}(\nu)=\hat{D}_{N}(\nu)$.

Proof. Let

$$
R(\alpha, \beta)=\frac{A([\alpha: \beta] ; \nu ; N)}{N}-\{\beta-\alpha\}
$$

Clearly 


$$
\hat{D}_{N}(\nu)=\sup \left(\sup _{0 \leq \alpha, \beta<1} R(\alpha, \beta), \sup _{0 \leq \alpha, \beta<1}-R(\alpha, \beta)\right) .
$$

But $-R(\alpha, \beta) \leq R(\beta, \alpha)$ since $A([\alpha: \beta] ; \nu ; N)+A([\beta: \alpha] ; \nu ; N) \geq N$ and $\{-x\}=1-\{x\}$ for real $x$. Hence

$$
\hat{D}_{N}(\nu)=\sup _{0 \leq \alpha, \beta<1}\left(\frac{A([\alpha: \beta] ; \nu ; N)}{N}-\{\beta-\alpha\}\right) \text {, }
$$

or

$$
\hat{D}_{N}(\nu)=\sup \left(\sup _{0 \leq \alpha \leq \beta<1} R(\alpha, \beta), \sup _{0 \leq \beta<\alpha<1} R(\alpha, \beta)\right)
$$

From (2.1),

$$
R(\alpha, \beta)= \begin{cases}\frac{A([\alpha, \beta] ; \nu ; N)}{N}-(\beta-\alpha), & 0 \leq \alpha \leq \beta \leq 1, \\ \alpha-\beta-\frac{A([\beta, \alpha] ; \nu ; N)}{N}, & 0 \leq \beta<\alpha \leq 1 .\end{cases}
$$

Replacing $R(\alpha, \beta)$ in (2.5) by this expression completes the proof.

Thus if the sequence is represented on the unit interval or the circle of unit circumference, the measure of discrepancy is the same.

\section{A relation between $D_{N}^{*}(\nu)$ and $D_{N}(\nu)$}

The following proposition relates the two functions $D_{N}^{*}(\nu)$ and $D_{N}(\nu)$

PROPOSITION. $D_{N}(\nu)=D_{N}^{*}(\nu)+\inf \left(D_{N}^{+}(v), D_{N}^{-}(v)\right)$, where

$$
\begin{aligned}
& D_{N}^{+}(\nu)=\sup _{0 \leq \beta<1}\left(\frac{A([0, \beta] ; \nu ; N)}{N}-\beta\right), \\
& D_{N}^{-}(\nu)=\sup _{0 \leq \beta<1}\left(\beta-\frac{A([0, \beta] ; \nu ; N)}{N}\right), \\
& D_{N}^{*}(\nu)=\sup \left(D_{N}^{+}(\nu), D_{N}^{-}(\nu)\right) .
\end{aligned}
$$

Proof. We need only show that $D_{N}(\nu)=D_{N}^{+}(\nu)+D_{N}^{-}(\nu)$. Without loss of generality assume that the elements $x_{j}, 1 \leq j \leq N$, are arranged in ascending order of magnitude. For notational convenience, let $x_{0}=0$ and 
$x_{N+1}=1$. Then the numbers $x_{0}, x_{1}, \ldots, x_{N+1}$ partition the unit interval so that

$$
D_{N}^{+}(\nu)=\sup _{\substack{x_{j} \leq \beta<x_{j+1} \\ j=0,1,2, \ldots, N}}\left(\frac{A([0, \beta] ; \nu ; N)}{N}-\beta\right) .
$$

Evaluating the supremum over each sub-interval $\left[x_{j}, x_{j+1}\right)$ gives

$$
D_{N}^{+}(\nu)=\sup _{j=1,2, \ldots, N}\left(\frac{j}{N}-x_{j}\right) \text {. }
$$

Similarly

$$
\overline{D_{N}^{-}}(\nu)=\sup _{j=1,2, \ldots, N}\left(x_{j}-\frac{j-1}{N}\right) .
$$

From (2.4) and the fact that $R(\alpha, \beta) \leq R\left(x_{i}, x_{j}\right)$ where $x_{i} \leq \alpha<x_{i+1}, x_{j} \leq \beta<x_{j+1}$ (for suitable $i$ and $j$ ) yields

$$
D_{N}(\nu)=\sup _{0 \leq i, j \leq N}\left(\frac{A\left(\left[x_{i}: x_{j}\right] ; \nu ; N\right)}{N}-\left\{x_{j}-x_{i}\right\}\right) \text {. }
$$

Alternatively

$$
\begin{aligned}
D_{N}(\nu) & =\sup _{0 \leq i, j \leq N}\left(\frac{j-i+1}{N}-x_{j}+x_{i}\right) \\
& =\sup _{0 \leq j \leq N}\left(\frac{j}{N}-x_{j}\right)+\sup _{0 \leq i \leq N}\left(x_{i}-\frac{i-1}{N}\right) \\
& =D_{N}^{+}(\nu)+D_{N}^{-}(\nu) .
\end{aligned}
$$

This follows from (3.1) and (3.2).

\section{The sequence formed from multiples of $\xi$}

We now turn to evaluating the discrepancies $D_{N}^{*}(\omega)$ and $D_{N}(\omega)$ for some particular values of $N$ related to the simple continued fraction expansion of $\xi$, where $\omega$ is the sequence of fractional parts of consecutive non-negative integer multiples of the irrational number $\xi$. The following notation is used. Write $t_{0}=\xi$ and express (for $n=0,1,2, \ldots)$, 


$$
\begin{aligned}
a_{n} & =\left[t_{n}\right], \\
t_{n+1} & =\frac{1}{\left\{t_{n}\right\}},
\end{aligned}
$$

where [ ] is the truncation operator.

In this way the continued fraction expansion of $\xi$ is identified by the expression

$$
\begin{aligned}
\xi & =a_{0}+\frac{1}{a_{1}+\frac{1}{a_{2}+\frac{1}{a_{3}+\ldots}}} \\
& =\left\{a_{0} ; a_{1}, a_{2}, a_{3}, \ldots\right\} .
\end{aligned}
$$

The partial convergents to $\xi$ are defined as

$$
\frac{p_{n+1, i}}{q_{n+1, i}}=\left\{a_{0} ; a_{1}, a_{2}, \ldots, a_{n}, i\right\}, i=1,2, \ldots, a_{n+1} \text {. }
$$

Note that

$$
\begin{aligned}
& \frac{p_{n+1, i}}{q_{n+1, i}}=\frac{p_{n-1}+i p_{n}}{q_{n-1}+i q_{n}}, p_{-2}=q_{-1}=0, q_{-2}=p_{-1}=1, \\
& \frac{p_{n+1, k}}{q_{n+1, k}}=\frac{p_{n+1}}{q_{n+1}},
\end{aligned}
$$

where $k=a_{n+1}$.

The total convergents $p_{n} / q_{n}, n=0,1,2, \ldots$, are important in Diophantine approximation theory since they provide the unique sequence of best rational approximations to $\xi$ in the sense that

$$
\left\|q_{n} \xi\right\|<\|q \xi\|, \quad 0<q<q_{n+1}, \quad q \neq q_{n},
$$

where

$$
\|q \xi\|=|q \xi-p|, p=\left[q \xi+\frac{1}{2}\right] \text {. }
$$

(That is, $\|q \xi\|$ is equal to the absolute difference between $q \xi$ and its nearest integer. Note that $\left.p_{n}=\left[q_{n} \xi+\frac{1}{2}\right].\right)$ we quote some results from the theory of continued fractions which we will need later: 


$$
\begin{aligned}
q_{n} p_{n+1, i}-p_{n} q_{n+1, i} & =(-1)^{n} ; \\
q_{n+1, i}\left\|q_{n} \xi\right\|+q_{n}\left\|q_{n+1, i} \xi\right\| & =1 ; \\
p_{n+1, i}\left\|q_{n} \xi\right\|+p_{n}\left\|q_{n+1, i} \xi\right\| & =\xi .
\end{aligned}
$$

\section{The three gap theorem (the Steinhaus conjecture)}

This theorem, originally conjectured by $H$. Steinhaus states that any $N$ consecutive elements of $\omega$ partition $C$ (or the unit interval) into sub-intervals or gaps of at most three different lengths and at least two if $\xi$ is irrational. Various proofs have appeared in the literature (see, for example, references [1], [5]-[9]). The theorem is also related to the ordering of the first $N$ elements of $\omega$. Let $\left(\left\{u_{j} \xi_{j}\right\}\right)$, $j=1,2, \ldots, N$, be that ordered sequence. That is, $\left\{u_{1}, u_{2}, \ldots, u_{N}\right\}=\{0,1, \ldots, N-1\}$ where $\left\{u_{j} \xi\right\}<\left\{u_{j+1} \xi\right\}$. It may be found from references [5] and [6] that the elements $u_{j}$ are obtained by the following relation,

$$
u_{j+1}= \begin{cases}u_{j}+u_{2}, & 0 \leq u_{j} \leq N-u_{2}, \\ u_{j}+u_{2}-u_{N}, & N-u_{2}<u_{j}<u_{N}, \\ u_{j}-u_{N}, & u_{N} \leq u_{j} \leq N,\end{cases}
$$

for $j=1,2, \ldots, N, u_{1}=0$, where

$$
\left\{\begin{array}{l}
u_{2}=\left\{\begin{array}{lll}
q_{n}, & n & \text { even } \\
q_{n+1, i-1}, & n & \text { odd },
\end{array}\right. \\
u_{N}=\left\{\begin{array}{lll}
q_{n+1, i-1}, & n \text { even, } \\
q_{n}, & n \text { odd, }
\end{array}\right.
\end{array}\right.
$$

which holds for $q_{n+1, i-1}<N \leq q_{n+1, i}, 2 \leq i \leq a_{n+1} \quad(n \geq 1)$.

For $q_{n}<N \leq q_{n+1, i} \quad(n \geq 1)$, 


$$
\left\{\begin{array}{l}
u_{2}=\left\{\begin{array}{lll}
q_{n}, & n & \text { even }, \\
q_{n-1}, & n & \text { odd },
\end{array}\right. \\
u_{N}=\left\{\begin{array}{lll}
q_{n-1}, & n & \text { even } \\
q_{n}, & n & \text { odd }
\end{array}\right.
\end{array}\right.
$$

THEOREM. The first $u_{2}+u_{N}=q_{n+1, i}\left(i=1,2, \ldots, a_{n+1}, n \geq 1\right)$ elements of $w$ partition the circle into gaps of two different lengths. In this case

$$
\begin{aligned}
& D_{q_{n+1, i}^{*}}^{(\omega)}= \begin{cases}\frac{1}{q_{n+1, i}}+\left(q_{n+1, i}-1\right)\left(\frac{p_{n+1, i}}{q_{n+1, i}}-\xi\right), & n \text { even, } \\
\sup \left(\frac{1}{q_{n+1, i}},\left(q_{n+1, i}-1\right)\left(\xi-\frac{p_{n+1, i}}{q_{n+1, i}}\right)\right), & n \text { odd, }\end{cases} \\
& D_{q_{n+1, i}}(\omega)=\frac{1}{q_{n+1, i}}+\left(q_{n+1, i}-1\right)\left|\frac{p_{n+1, i}}{q_{n+1, i}}-\xi\right| \text {. }
\end{aligned}
$$

Proof. With $N=u_{2}+u_{N}=q_{n+1, i},(5.1)$ becomes

$$
u_{j+1}= \begin{cases}u_{j}+u_{2}, & 0 \leq u_{j}<u_{N}, \\ u_{j}-u_{N}, & u_{N} \leq u_{j} \leq N,\end{cases}
$$

for $j=1,2, \ldots, N\left(u_{1}=0\right)$. Hence, for this value of $N$, there are only two gap lengths $\left\|u_{2} \xi\right\|$ and $\left\|u_{N} \xi\right\|$. From (5.1) note that if $N \neq u_{1}+u_{2}$, the circle is partitioned into gaps of three different lengths.

$$
(5.4) \text { is equivalent to }
$$

$$
u_{j}=\left((j-1) u_{2}\right) \bmod N, j=1,2, \ldots, N \text {. }
$$

Substituting (5.2) and (5.3) into (5.5) yields

$$
u_{j}=\left((-1)^{n}(j-1) q_{n}\right) \bmod q_{n+1, i}, j=1,2, \ldots, q_{n+1, i} .
$$

It is seen that there exists a non-negative integer $k$ so that 


$$
u_{j}=(-1)^{n}(j-1) q_{n}-(-1)^{n} k q_{n+1, i}
$$

Solving this linear Diophantine equation by use of (4.1) yields

$$
j-1=q_{n+1, i}\left\{\frac{u_{j} p_{n+1, i}}{q_{n+1, i}}\right\} \text {, }
$$

$$
k=u_{j} p_{n}-\left[\frac{u_{j} p_{n+1, i}}{q_{n+1, i}}\right] q_{n} .
$$

From (5.7) it follows that

$$
\left\{u_{j} \xi\right\}=(j-1)\left\|q_{n} \xi\right\|+k\left\|q_{n+1, i} \xi\right\|
$$

Substituting expressions for $j$ and $k$ from (5.8), (5.9) and using (4.2) and (4.3) yields

$$
\left\{u_{j} \xi\right\}=u_{j} \xi-\left[\frac{u_{j} p_{n+1, i}}{q_{n+1, i}}\right] .
$$

That is

$$
\left[u_{j} \vec{\xi}\right]=\left[\frac{u_{j} p_{n+1, i}}{q_{n+1, i}}\right] .
$$

Substitution of (5.8) into (3.2) with the inclusion of (5.11) yields

$$
\begin{aligned}
\vec{D}_{q_{n+1, i}}(\omega) & =\sup _{j=1,2, \ldots, q_{n+1, i}} u_{j}\left(\xi-\frac{p_{n+1, i}}{q_{n+1, i}}\right) \\
& =\sup _{u_{j}=0,1, \ldots, q_{n+1, i}-1} u_{j}\left(\xi-\frac{p_{n+1, i}}{q_{n+1, i}}\right) \\
& =\left\{\begin{array}{l}
0 \text { even, } \\
\left(q_{n+1, i}-1\right)\left(\xi-\frac{p_{n+1, i}}{q_{n+1, i}}\right), n \text { odd. }
\end{array}\right.
\end{aligned}
$$

This follows from the fact that $\xi-p_{n+1, i} / q_{n+1, i}$ is negative for even $n$ and positive otherwise.

To determine $D_{q_{n+1, i}^{+}}^{(\omega)}$ note that 


$$
D_{q_{n+1, i}^{+}}^{(\omega)}=\frac{1}{q_{n+1, i}}-\underset{j=1,2, \ldots, q_{n+1, i}}{\inf }\left(\left\{u_{j} \xi\right\}-\frac{j-1}{q_{n+1, i}}\right) .
$$

Following the same procedure as above, it is found that

$$
D_{q_{n+1, i}^{+}}^{(\omega)}= \begin{cases}\frac{1}{q_{n+1, i}}+\left(q_{n+1, i}-1\right)\left(\frac{p_{n+1, i}}{q_{n+1, i}}-\xi\right), & n \text { even, } \\ \frac{1}{q_{n+1, i}}, & n \text { odd. }\end{cases}
$$

The theorem now follows from the proposition.

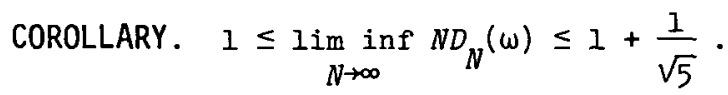

Proof. The lower bound is easily found. (See Kuipers and Neiderreiter [4], page 90.) For the upper bound, first note that

$$
\lim _{N \rightarrow \infty} \inf N D_{N}(\omega) \leq \underset{n \rightarrow \infty}{\lim \inf } q_{n} D_{q_{n}}(\omega) .
$$

From the theorem,

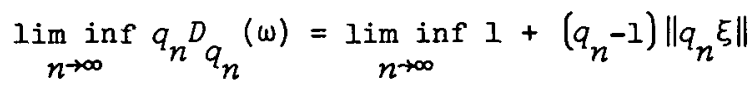

$$
\begin{aligned}
& =1+\lim _{n \rightarrow \infty} \inf q_{n}\left\|q_{n} \xi\right\| \text {. }
\end{aligned}
$$

From a theorem of Hurwitz (see, for example, Hardy and Wright [2], Theorem 194),

$$
\begin{aligned}
\sup _{\xi} \operatorname{Iim} \inf q\|q \xi\| & =\sup _{q \rightarrow \infty} \lim \inf _{n \rightarrow \infty} q_{n}\left\|q_{n} \xi\right\| \\
& =\frac{1}{\sqrt{5}} .
\end{aligned}
$$

The supremum occurs at all values of $\xi$ which have $t_{j}=(1+\sqrt{5}) / 2$ for some non-negative integer $j$.

Thus the corollary follows. 


\section{References}

[1] J.H. Halton, "The distribution of the sequence $\{n \xi\}$ $(n=0,1,2, \ldots)$ ", Proc. Cambridge Philos. Soc. 61 (1965), $665-670$.

[2] G.H. Hardy and E.M. Wright, An introduction to the theory of numbers, 4th ed. (Clarendon Press, Oxford, 1960; reprinted 1971).

[3] A.Y. Khintchine, Continued fractions (translated by P. Wynn. Noordhoff, Groningen, 1963).

[4] L. Kuipers and $H$. Neiderreiter, Uniform distribution of sequences (John Wiley and Sons, New York, London, 1974).

[5] A. Révuz, "Sur la repartition des points $e^{v i \theta}$ ", C.R. Acad. Sci. 228 (1949), 1966-1967.

[6] N.B. Slater, "Gaps and steps for the sequence $n \theta \bmod 1$ ", Proc. Cambridge Phizos. Soc. 63 (1967), 1115-1122.

[7] V.T. Sós, "On the theory of Diophantine approximations, I", Acta Math. Acad. Sci. Hungar. 8 (1957), 461-472.

[8] V.T. Sós, "On the distribution mod 1 of the sequence na ", Ann. Univ. Sci. Pudapest. Ë̈tvös Sect. Math. 1 (1958), 127-134.

[9] S. Swierckowski, "On successive settings of an arc on the circumference of a circle", Fund. Math. 46 (1958), 187-189.

Department of Mathematics, University of Wol longong, Woll longong, New South Wales 2500, Australia. 